\title{
Narrative review of ring fixator management of recurrent club foot deformity
}

\author{
Zachery Meyer ${ }^{1}$, Jacob R. Zide ${ }^{2}$, Alexander Cherkashin ${ }^{1} \wedge$, Mikhail Samchukov ${ }^{1}$, Daniel D. Bohl ${ }^{3}$, \\ Anthony I. Riccio ${ }^{1 \wedge}$
}

${ }^{1}$ Department of Orthopaedic Surgery, Texas Scottish Rite Hospital, Dallas, TX, USA; ${ }^{2}$ Department of Orthopaedic Surgery, Baylor University Medical Center, Dallas, TX, USA; ${ }^{3}$ Baylor University Medical Center, Department of Orthopaedic Surgery, Dallas, TX, USA

Contributions: (I) Conception and design: AI Riccio, M Samchukov, A Cherkashin; (II) Administrative support: None; (III) Provision of study materials or patients: None; (IV) Collection and assembly of data: None; (V) Data analysis and interpretation: None; (VI) Manuscript writing: All authors; (VII) Final approval of manuscript: All authors.

Correspondence to: Anthony I. Riccio, MD. Texas Scottish Rite Hospital for Children, 2222 Welborn Street, Dallas, TX 75219, USA.

Email: Anthony.Riccio@tsrh.org.

\begin{abstract}
Despite the widespread use of the Ponseti method for treatment of clubfeet, there continue to be a significant number of patients who present with a severe, stiff clubfoot as a result of extensive intraarticular soft tissue release or lack of access to care. In such patients, circular external fixators can be utilized for deformity correction with distraction across soft tissues, joints, and osteotomies. Ilizarov or hexapod circular fixators may be utilized according to surgeon preference. Indications for soft tissue release and osteotomies to aid in correction of clubfoot deformity with Ilizarov and hexapod fixators are not standardized and are guided by patient age, joint congruity, soft tissue suppleness, and osseous deformity. Correction time varies according to clubfoot deformity severity. Following deformity correction, external fixators are left in place for several weeks to stabilize the soft tissues and allow for osteotomy healing. Complications range from relatively minor pin tract infections that resolve with oral antibiotics to tarsal tunnel syndrome, osteomyelitis, or disabling arthritis requiring revision procedures. At Scottish Rite Hospital for Children, we prefer to correct severe residual clubfoot deformity with a hexapod external fixator. Acute correction and gradual correction via distraction are considered for each segmental deformity and utilized to efficiently correct deformity while minimizing soft tissue trauma. The purpose of this article is to summarize the relevant literature related to circular external fixator treatment of recurrent clubfoot deformity and outline our approach to the segmental deformities of the foot and ankle in this patient population.
\end{abstract}

Keywords: Clubfoot; ring fixation; deformity; multiplanar fixation; Ilizarov

Submitted Nov 24, 2020. Accepted for publication Mar 25, 2021.

doi: $10.21037 /$ atm-20-7621

View this article at: http://dx.doi.org/10.21037/atm-20-7621

\section{Introduction}

Few pediatric foot pathologies are as daunting as the multiply operated clubfoot with residual or recurrent deformity. Such feet are usually stiff from surgical scarring, frequently have some degree of soft tissue envelope compromise and often have altered articular morphology or frank degenerative disease of one or more joints from prior intra-articular surgery. While worldwide adoption of the Ponseti method has led to improved clubfoot correction with less need for extensive circumferential surgery, posteromedial capsular release is still performed for resistant, untreated and some relapsed infantile clubfoot

^ ORCID: Alexander Cherkashin, 0000-0002-0422-9158; Anthony I. Riccio, 0000-0002-8386-9312; Mikhail Samchukov, 0000-0002-3972-0828. 
deformities. Permanent alterations in tibiotalar and talonavicular morphology along with surgical scarring from such intra-articular procedures can make management of residual or recurrent deformity difficult. Moreover, such feet tend to be significantly foreshortened, which can limit acute correction options for concerns of creating an even shorter and potentially nonfunctional foot.

Gradual correction of clubfoot deformities with Ilizarov and hexapod circular external fixators is an effective approach when acute correction is not desirable or possible. Ilizarov discovered that gradual distraction stimulates growth and maintenance of tissues, a principle he described as "the law of tension stress $(1,2)$." Complex clubfoot deformity can be corrected with directed distraction across the contracted soft tissues and joints or with distraction across osteotomies when deformities are fixed, joints are degenerated, or abnormal fusions are present (3). In the following section we review the literature regarding circular external fixator treatment of clubfoot deformity, including circular fixator configurations, soft tissue releases and osteotomies, functional outcome and correction results, and complications. We conclude with an outline of our approach to the segmental deformities present in the recurrent clubfoot, which includes our indications for circular fixator treatment and fixator configurations, acute and gradual correction techniques, and case examples. We present the following article in accordance with the Narrative Review reporting checklist (available at http://dx.doi.org/10.21037/ atm-20-7621).

\section{Methods}

To identify articles pertinent to this narrative review, PubMed was searched using the terms "clubfoot", "ring fixator", "Ilizarov," "gradual correction," talipes equinovarus," distraction osteogenesis," and "foot deformity" in various combinations from 1980 through September 2020. Review articles and original scientific papers written in English with a minimum of level IV evidence were included.

\section{Discussion}

\section{Fixator configurations}

Ilizarov and hexapod external fixators provide a versatile approach for the correction of complex foot and ankle deformities (4). The decision to use an Ilizarov or hexapod circular fixator is determined predominantly by surgeon preference. While specific details of frame assembly and fixation vary depending on the deformity, osteotomy location, and method of correction, some general guidelines of frame assembly are available in the literature. For clubfoot correction utilizing Ilizarov type external fixators, the standard frame consists of two tibial rings secured with crossing tensioned fine wires and/or half pins, a forefoot half ring secured with tensioned olive wires traversing the first and fifth metatarsal necks, and calcaneal half ring secured with two oppositely directed tensioned olive wires through the calcaneal tuberosity $(3,5-10)$. In pediatric patients, an additional tensioned wire is often placed through the distal tibial epiphysis and secured to the tibial ring to prevent epiphysiolysis during correction (11). Tibial fine wires are tensioned at 110-130 kilograms of force and foot wires are tensioned at 90-100 kilograms of force (7). Tibial, calcaneal, and forefoot rings are connected with threaded rods and hinges to allow independent correction of the hindfoot, midfoot, and forefoot deformities (Figure 1). Tensioned olive wires placed from lateral to medial through the talar neck and from medial to lateral through the navicular can be utilized to reduce the talonavicular subluxation often seen in clubfoot deformities $(5,11)$. In general, equinus can be corrected by distraction of tibial-calcaneal rods and shortening of tibial-forefoot rods, hindfoot varus can be corrected by differential distraction between medial and lateral tibial-calcaneal rods, and adductus and cavus can be corrected by differential distraction between the medial and lateral calcaneal-forefoot rods $(3,6-8,12,13)$.

Hexapod circular fixators rely on six interconnecting struts arranged between rings to create a parallel kinematic system that allows for simultaneous deformity correction in three planes $(4,11)$. Hexapod circular frame configuration for foot deformity is therefore dependent on the foot segment or segments in need of correction. Rings are secured to the tibia, calcaneus, and forefoot in a similar manner to Ilizarov fixators. The rings are connected by struts that allow correction between the foot segments. Taylor introduced carpentry terms to describe hexapod frame configurations. Miter frames allow for simultaneous and independent correction of the hindfoot and mid/ forefoot relative to the tibia and butt frames allow for correction of $\mathrm{mid} /$ forefoot deformities relative to the hindfoot and tibia (Figure 2). Cherkashin et al. introduced a comprehensive scheme for the description of hexapod fixators, however, to date, there is no universally accepted nomenclature (4). 

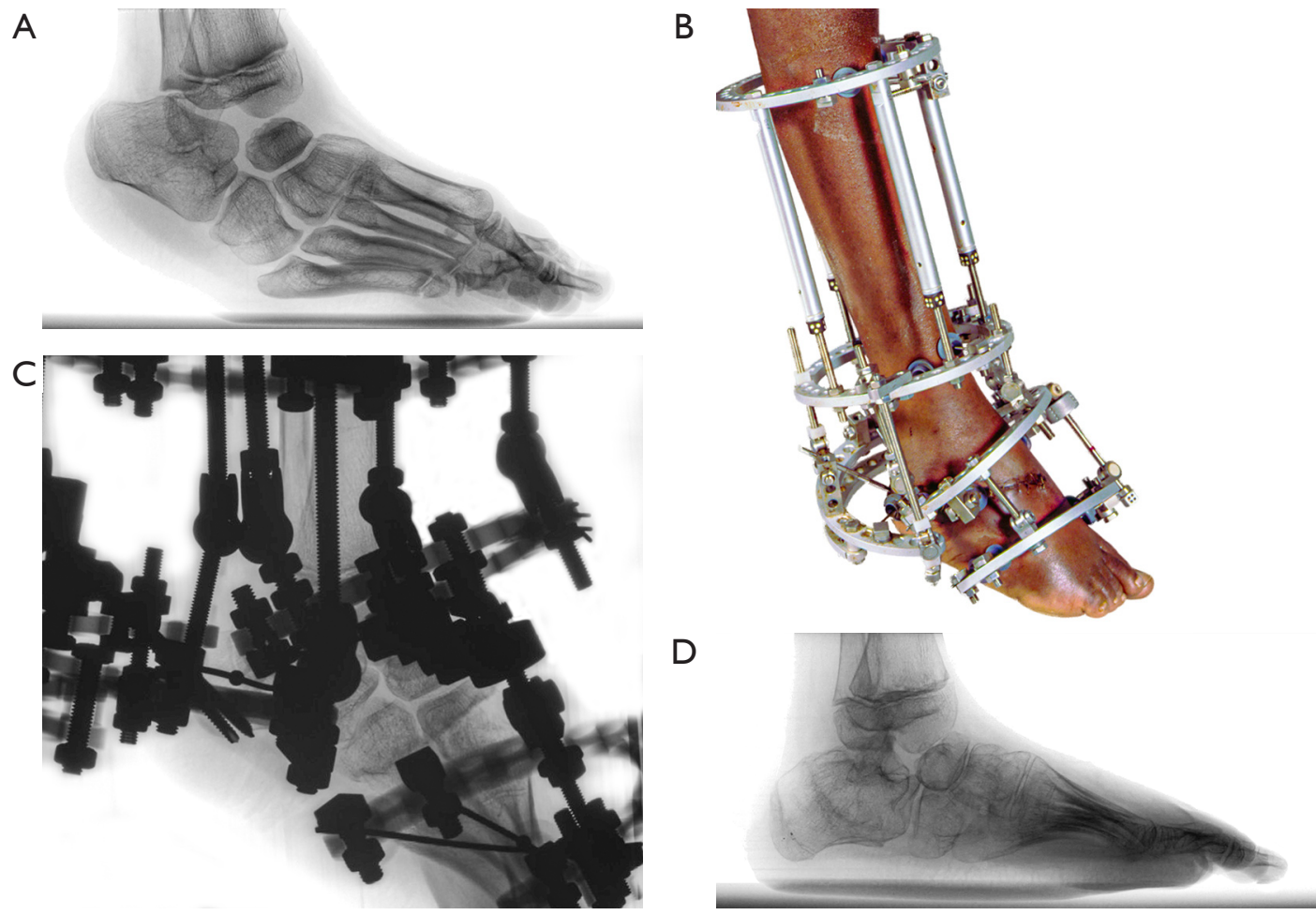

Figure 1 Gradual recurrent clubfoot deformity correction with hindfoot and midfoot lengthening after multiple prior surgeries including a talectomy. (A) Lateral radiograph before correction; (B) external fixator miter assembly for independent hindfoot and midfoot correction through a 'V'-osteotomy; (C) lateral radiograph during correction through a 'V'-osteotomy; (D) lateral radiograph after correction (note the increase in foot length).

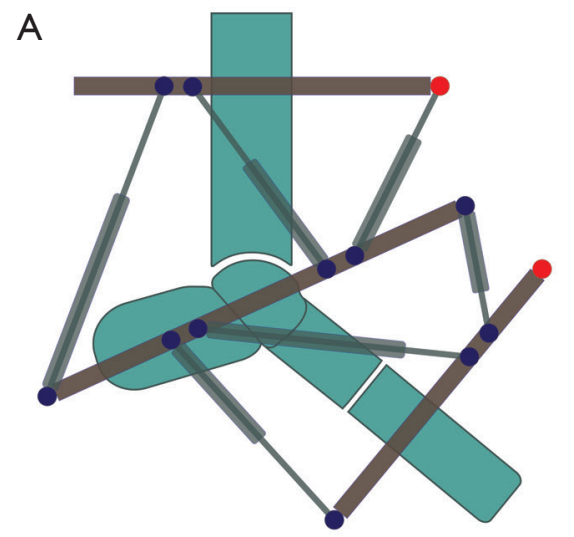

B

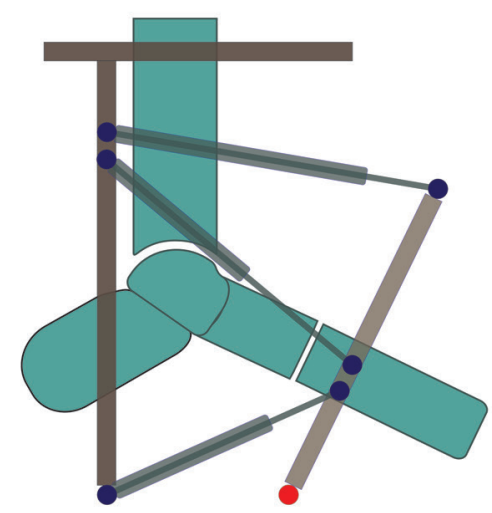

Figure 2 Hexapod foot frame assemblies. (A) Miter frame for hindfoot and midfoot correction; (B) Butt frame assembly for correction of midfoot deformity.

\section{Soft tissue releases and osteotomies}

Indications for soft tissue release and osteotomies to aid in correction of clubfoot deformity with Ilizarov and hexapod fixators are not well established. Paley outlined guiding principles for the correction of complex foot deformities using Ilizarov fixators: (I) soft tissue and joint distraction without 
osteotomies can correct deformity when joints are congruent (or brought into a congruent position) and there is no fixed bony deformity. (II) Osteotomies are necessary when there is fixed bony deformity, incongruent joint relationships, previous fusions or nonunions, or a neuromuscular imbalance is present that may preclude maintenance of correction with soft tissue procedures alone. Exceptions to the second principle may be made for patients younger than 8 years of age, who possess significant bony remodeling potential (3). Gupta et al. reported good or excellent results, based on the International Clubfoot Study Group (ICFSG) scoring system, in children with relapsed clubfeet treated with an Ilizarov fixator and soft tissue distraction alone. Prem et al. reported similarly good outcomes and found that deformity correction following Ilizarov treatment was maintained 5 years after the index surgery (14). While these principles are generally accepted, Hosny demonstrated soft tissue distraction alone may have a role in older patients with stiff deformities. He reported good results, defined as painless plantigrade feet, in 13/14 relapsed clubfeet in pediatric and adult patients treated with gradual correction in an Ilizarov fixator without soft tissue release or osteotomy (8). When soft tissue releases or osteotomies are utilized, the extent of soft tissue release (percutaneous versus open plantar fascia release for cavus correction, tendo Achilles lengthening or tenotomy for equinus correction, or posteromedial release for equinocavovarus correction) and osteotomy approach (percutaneous or open midfoot osteotomy for cavus correction, percutaneous or open calcaneus or supramalleolar osteotomies for varus and equinus correction) varies in the current literature $(3,6-10,12,13,15,16)$.

\section{Correction and outcomes}

Reported correction times for clubfoot deformities in Ilizarov and hexapod fixators range from 27 to 30 days $(5,9,12)$, but ultimately depend on severity of deformity. Deformity correction is typically started two (10) or three (7) days after fixator application, but may be delayed as long as seven days (17). Correction occurs in 75 millimeter (mm) to $1 \mathrm{~mm}$ increments per day $(12,17)$. In general, following deformity correction external fixators are left in place to stabilize the corrected soft tissues and allow for osteotomy healing. During this time, patients are permitted to weight bear in the fixator. Bradish and Noor outline a typical treatment algorithm: stabilization of deformity correction with a fixator for six weeks, followed by fixator removal and short leg cast for six weeks, followed by ankle foot orthosis (AFO) use for six months (9).
Outcomes following circular fixator treatment of clubfoot deformities are difficult to compare due the heterogeneity in treatment approaches and lack of standard outcome measures. Grill and Franke obtained plantigrade feet in 9 pediatric patients ages 8 to 15 with relapsed or untreated clubfoot. They did not perform concomitant soft tissue release or osteotomies at the time of fixator application, though five patients required subsequent procedures after fixator removal. At 3.3-year follow-up, all patients were satisfied with the correction and able to wear standard shoes (12). Bradish and Noor treated 17 feet in 12 children with recurrent clubfoot with gradual soft tissue distraction. They noted 13 of 17 feet achieved an excellent or good result. All remaining patients with a poor or fair result experienced deformity recurrence. They recommended anterior tibialis tendon transfer to prevent recurrent deformity and maintain function (9). Lee $e t$ al. utilized an Ilizarov fixator to correct 12 recurrent arthrogypotic clubfeet and were able to obtain painless, plantigrade feet in 11 of 12 patients. Soft tissue procedures such as talonavicular capsulotomy, tendon Achilles lengthening, posterior tibialis tendon lengthening, and posterior capsulotomy were utilized at the time of fixator application (5). Eidelman et al., citing the steep learning curve required for expert Ilizarov technique execution, utilized the Taylor spatial frame to correct 10 relapsed arthrogrypotic clubfeet in seven pediatric patients. A standard frame, consisting of a foot " $U$ " plate and tibial ring connected by six struts, or a butt frame, consisting of a forefoot ring connected via 6 struts a " $U$ " plate secured to the tibial ring, were used depending on if a midfoot or forefoot correction was required. Gigli saw osteotomies were performed to facilitate correction and a plantigrade foot was achieved in all patients following treatment (17).

While these results and others are encouraging, Freedman et al. caution against unbridled optimism when utilizing circular fixators for the treatment of clubfoot. In their series of 21 previously operated on clubfeet with soft tissue release (tendon Achilles lengthening, posterior release, or posteromedial release) and Ilizarov distraction, $52.4 \%$ of patients experienced a poor result, defined as significant residual deformity, pain, and activity modifications at average follow-up of 6 years. Pain and limited function may have been caused by the soft tissue releases utilized, as posteromedial soft tissue release without Ilizarov distraction has been shown to cause stiff, poorly functional feet (18). Although stiffness following gradual correction of clubfeet has been reported elsewhere as well $(14,19,20)$, Gupta et al. postulated stiffness may be a result of previous open surgery rather than a complication of the 
Ilizarov technique (10).

\section{Complications}

While many authors have been able to correct complex foot deformity using circular external fixators, complications arising from these treatment modalities are numerous. Early complications include pin tract infections, soft tissue compromise, joint subluxation, flexion deformities of the toes, epiphysiolysis and premature consolidation of osteotomy sites. Pin tract infections are extremely common, the majority of which can be treated with oral antibiotics or pin removal $(5,6,8,10,14,17)$. Osteomyelitis may develop and can present even after frame removal (16). Soft tissue compromise from entrapment between frame components during correction (16) or from overzealous distraction (5) has been reported. Lamm et al. recommend routine decompression of the tarsal tunnel to avoid iatrogenic injury to the tibial nerve, which is particularly at risk during equinus correction (11). Wallander et al. reported distal tibial epiphysiolysis caused by distraction (20). This can be prevented by placing a tensioned fine wire through the epiphysis and securing it to the tibial ring (11). Flexion deformities of the toes can be prevented with physical therapy and by strapping the toes to the fixator during correction. Persistent toe deformity may require flexor tenotomy and percutaneous wire fixation for definitive correction (6). Late complications include unintended ankylosis, deformity recurrence, and degenerative joint changes. Ferreira et al. reported on 56-month follow-up of adolescent patients with relapsed clubfeet treated with a simplified Ilizarov fixator. While $77 \%$ of patients reported a good result, which was defined as a painless foot and ankle, patient capacity to walk on a plantigrade foot and wear conventional shoes, and the absence of significant recurrence for at least two years, the result was not durable in all $37 \%$ of patients in their cohort required a subsequent arthrodesis for disabling arthritis or recurrent deformity at an average of 21 months from Ilizarov treatment (7).

\section{Approach to circular fixator treatment of clubfeet at Scottish rite for children}

Successful management of the recurrent clubfoot is predicated on appropriate patient selection and accurate assessment of the segmental deformities of the foot and ankle. Given the duration of treatment and potential complications, the decision to proceed to circular fixator assisted correction of clubfoot deformity is a shared choice between the surgeon and the patient and made after extensive discussion. In general, we offer circular external fixator assisted correction of clubfeet to older children ( $>8$ years of age), adolescents, and young adult patients with severe, stiff deformities who have failed one or more attempts of treatment via the Ponseti method, and in patients who have undergone extensive or multiple soft tissue release with a nonpliable or compromised soft tissue envelope.

After the decision has been made to utilize circular external fixation, the ankle, hindfoot, midfoot, and forefoot deformities must be determined by their deviation from normal parameters and understood in relation to each other to develop an efficient and complete correction plan. We assess deformity with physical examination, radiographs, and gait analysis. We rarely utilize advanced imaging. If our initial estimation of deformity is inaccurate, we adjust our circular fixator prescription until an acceptable clinical result is achieved at the conclusion of strut turns. In the untreated or recurrent clubfoot, the relevant deformities are ankle equinus, hindfoot varus, midfoot cavus, and forefoot adductus. In this section discuss each segmental deformity separately and outline our considerations and approaches for gradual and acute correction of the relevant soft tissue and osseous structures.

\section{Ankle deformity}

Options for ankle deformity management include acute and gradual correction either through the tibiotalar joint or through the distal tibia itself. Though performed less commonly, relative correction of a calcaneus or equinus deformity can be achieved by altering the calcaneal-tibial relationship via a calcaneal tuberosity osteotomy (heel slide). This latter option is commonly reserved for cases in which tibiotalar degenerative changes, a poor soft tissue envelope or severely abnormal distal tibial deformity or bone quality preclude more proximal deformity correction.

\section{Acute soft tissue correction}

Residual equinus is the most commonly encountered ankle deformity in patients with residual clubfoot deformity. Under ideal circumstances, soft tissue procedures, such as open tendon Achilles lengthening or posterior tibiotalar capsular release, are utilized to correct the deformity. These procedures are our preferred means of correcting equinus in patients with normal talar dome morphology who have 

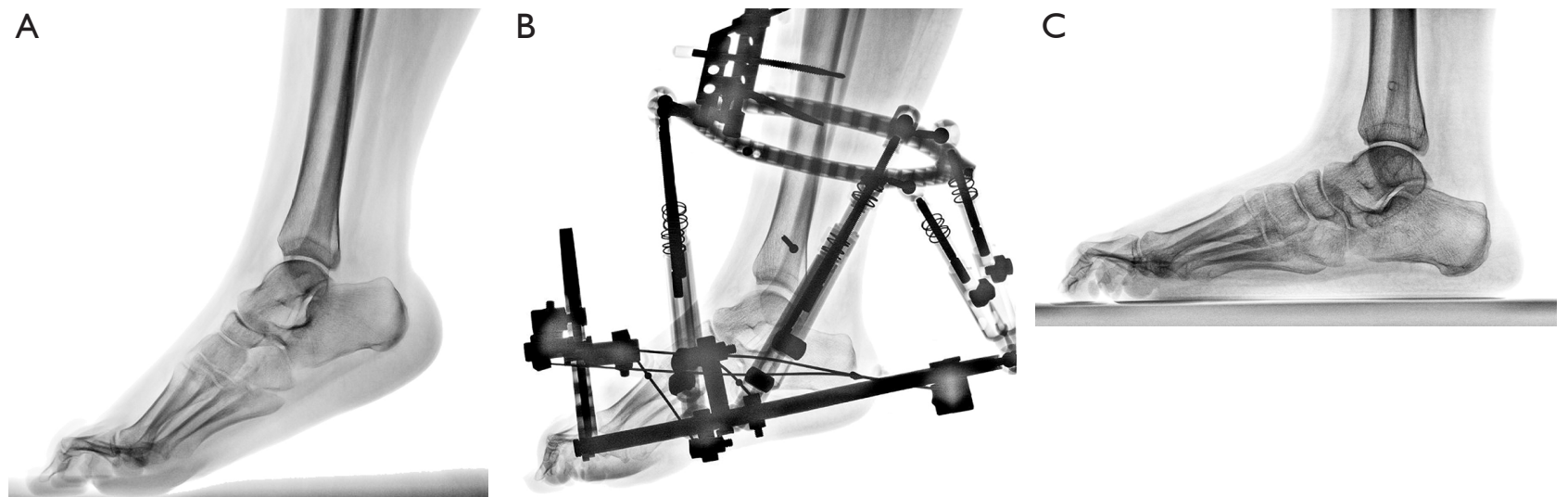

Figure 3 Gradual equinus correction through the tibiotalar joint. (A) Lateral standing radiographs demonstrate equinus and a congruent tibiotalar joint; (B) gradual equinus with hexapod frame; (C) standing radiographs 2 years after equinus correction.

not undergone prior intra-articular ankle surgery, but are less relied upon in patients with residual clubfoot deformity who have undergone prior intra-articular release. Following acute correction, the ankle can be maintained in dorsiflexion with fixation in a butt frame assembly. Acute equinus correction through the ankle joint is contraindicated in patients with limited or absent tibiotalar range of motion, global degenerative changes in the tibiotalar joint, tibiotalar joint incongruity, or associated distal tibial deformity requiring correction.

\section{Gradual soft tissue correction}

In patients who are not candidates for acute equinus correction or in those in whom acute correction was incomplete, gradual equinus correction through the tibiotalar joint may be considered. Gradual distraction across the tibiotalar joint permits stress relaxation of the surrounding tissues and subsequent gradual equinus correction. This technique requires a healthy, congruent tibiotalar articulation (Figure 3). As with acute correction, joint incongruency and global degenerative disease are contraindications for gradual correction, unless this technique is being used to position the ankle in anticipation of a planned tibiotalar arthrodesis. When performing gradual equinus correction with simultaneous correction of midfoot deformities, a miter frame assembly is required.

\section{Bony correction of equinus through the distal tibia}

Despite significant improvement of equinus deformity with soft tissue procedures, incomplete correction is frequently encountered, especially in patients who have previously been treated with extensive intra-articular release due to significant scarring. In such patients, bony correction of the residual equinus through the distal tibia is a reliable method to obtain a neutral ankle. Distal tibial osteotomy should also be considered in patients with distal tibial deformity. Options for bony correction include acute correction with either external or internal fixation as well as gradual correction using a multiplanar external fixator.

Acute correction requires an open approach to the distal tibia and fibula to ensure a properly positioned and executed osteotomy. Patients with isolated sagittal plane deformity without significant limb length difference are most easily treated with a sagittal dome osteotomy. Anterior closing wedge osteotomies are useful in the rare case the affected limb needs to be shortened to correct a limb length discrepancy. When lengthening and sagittal plane deformity correction are needed a transverse osteotomy with gradual correction should be considered. For patients with isolated coronal plane deformity, coronal dome, closing, or opening wedge osteotomies may be utilized depending on the need to address a limb length difference.

Acute correction may be maintained with a circular fixator or with internal fixation using a plate and screw construct. External fixation is ideal if the surgical plan involves correction of ankle deformity followed by gradual correction of a midfoot deformity. Correcting and maintaining the ankle in the desired position facilitates fine wire placement and determination of midfoot and hindfoot correction parameters. A butt frame is employed in such instances as it offers secure fixation of the distal tibia and fibula while allowing for gradual midfoot correction (Figure 4). Internal 
A

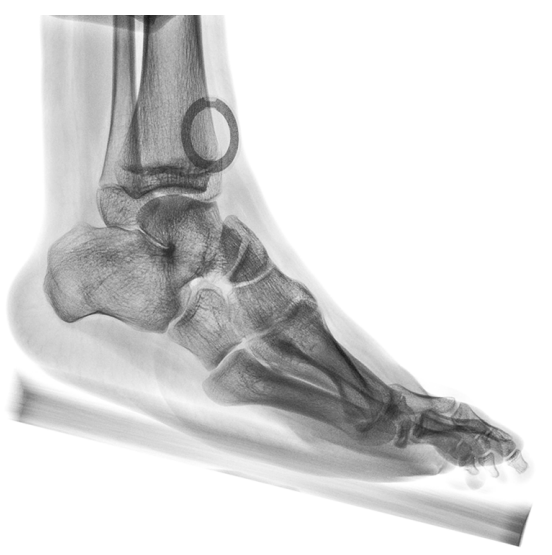

C

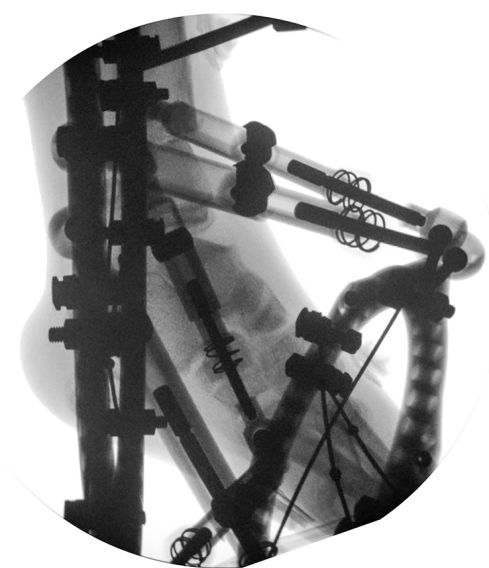

B
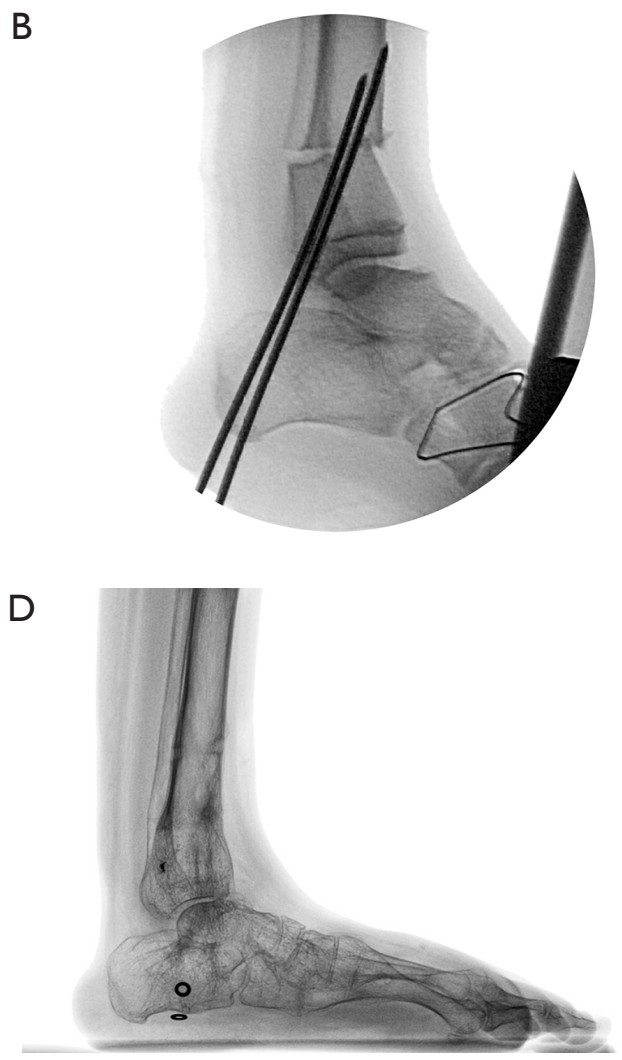

Figure 4 Acute equinus correction using a supramalleolar osteotomy incorporated into a butt frame to allow gradual midfoot correction. (A) Preoperative lateral radiographs demonstrate flattening of the talar dome limiting dorsiflexion; (B) intraoperative radiographs with temporary extra-articular fixation of the supramalleolar osteotomy; (C) following acute correction, the distal tibia is incorporated into the butt frame allowing for gradual midfoot cavus correction; (D) lateral radiograph of the result following frame removal.

fixation of a distal tibia osteotomy is also a useful option in patients who require residual equinus correction after midfoot cavus correction. In such cases, it is advisable to delay the osteotomy until after frame removal, initiation of weightbearing and resolution of osteopenia (Figure 5).

\section{Relative correction of equinus via calcaneal osteotomy}

Though less commonly performed, relative correction of equinus can be achieved by performing a calcaneal tuberosity osteotomy and sliding the proximal segment plantarward. In such cases, the tibiotalar joint is left in equinus and clinical correction is achieved by establishing a more normal relationship between the posterior calcaneus and the tibia. This technique is particularly useful in patients with a varus hindfoot deformity as both deformities can be addressed simultaneously. If acute correction is planned, stabilization is achieved using a butt frame assembly. If gradual correction is desired due to concerns of limited soft tissue compliance, two options are available. The calcaneal tuberosity fragment may be secured to a ring and translated using a miter frame to allow for concomitant midfoot deformity correction. As an alternative, midfoot correction can be achieved first using a butt frame assembly followed by frame revision and hindfoot correction.

\section{Staged correction of equinus}

Lastly, it is critical to consider the possibility that even when acute correction is attempted, incomplete correction due to a lack of soft tissue compliance is commonly encountered. Even when desired sagittal plane ankle deformity correction 
A

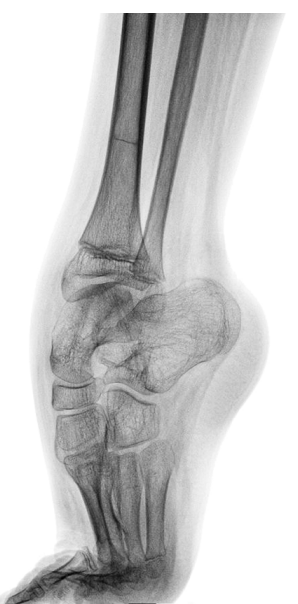

B

$\mathrm{D}$

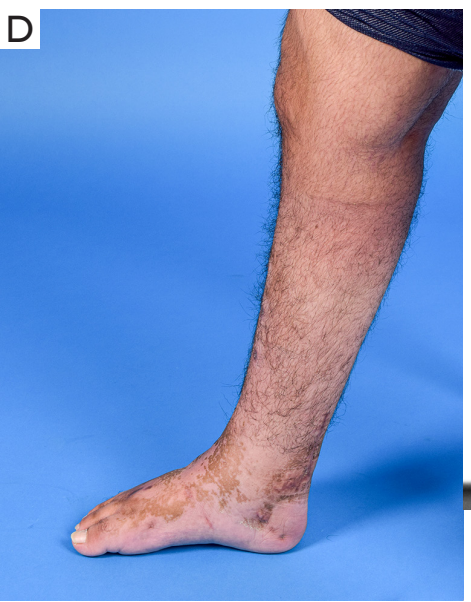

G

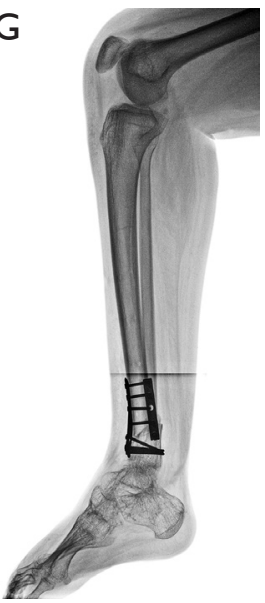

$\mathrm{H}$
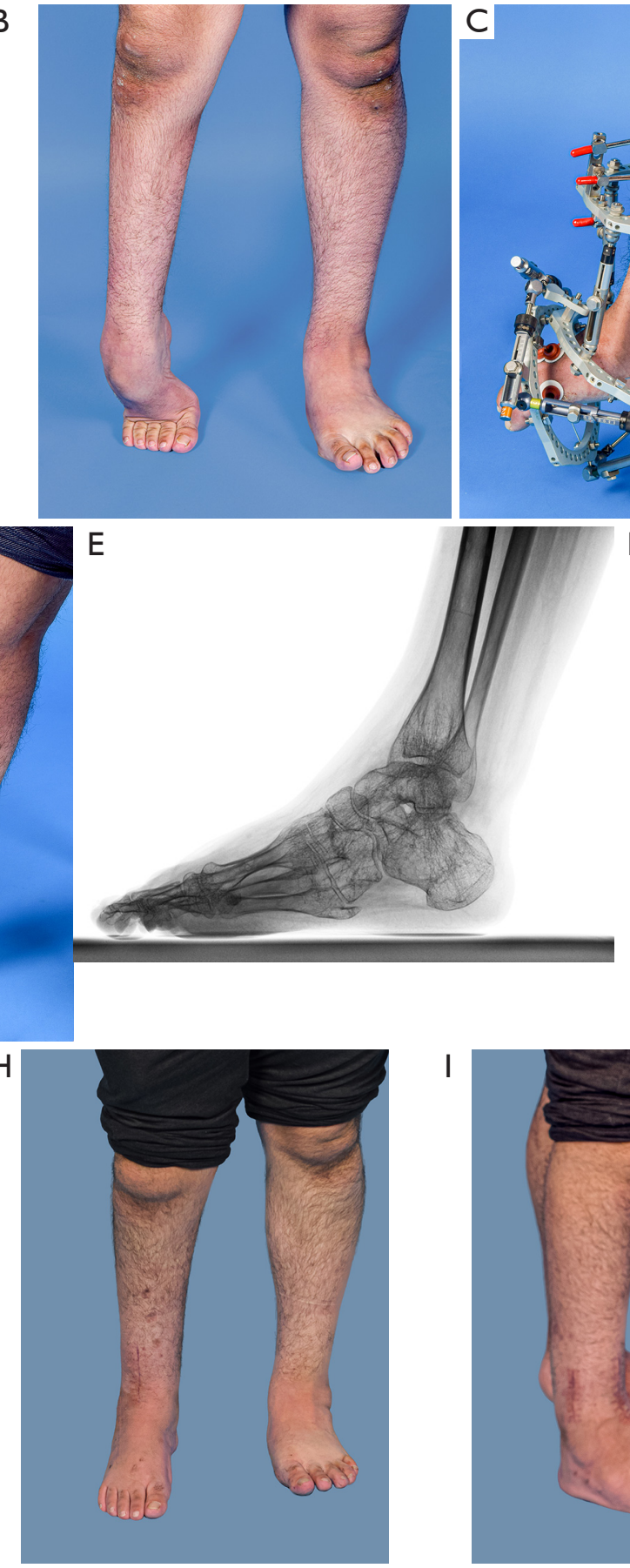

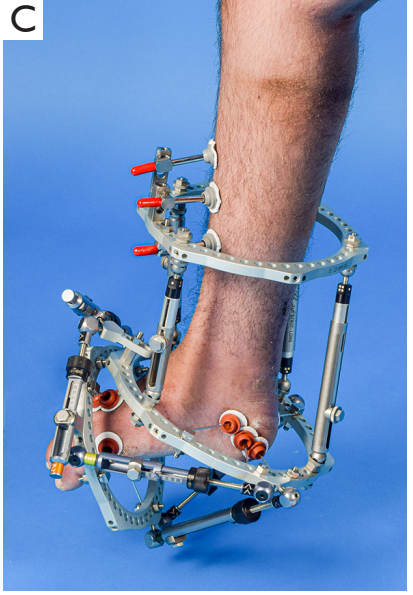

$\mathrm{F}$

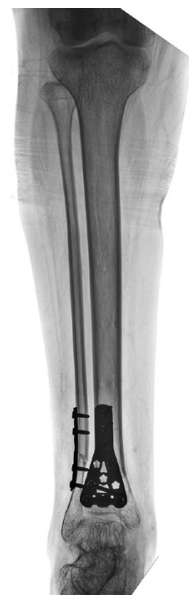

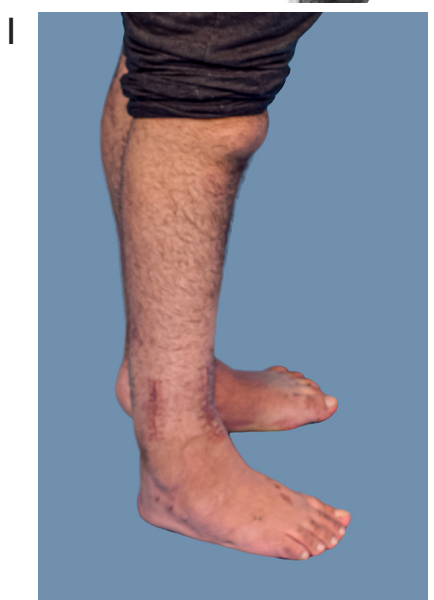

Figure 5 Staged correction of a severe clubfoot deformity in a 14-year-old male. (A) Preoperative lateral standing radiograph; (B) preoperative appearance of the foot demonstrating the equino-cavo-varus deformity; (C) clinical photograph during gradual cavus correction after partial equinus correction; (D,E) clinical and radiographic images illustrating midfoot deformity correction with residual equinus; (F,G) radiographs after subsequent acute correction using a supramalleolar osteotomy with internal fixation; (H,I) clinical photographs following treatment. 
is achieved, subsequent or simultaneous gradual cavus correction may create increased tension across the hindfoot resulting in some loss of correction following frame removal. In patients with both severe equinus and cavus deformities, an inability to obtain full cavus correction may result in ongoing increased forefoot pressure during ambulation and an inability to load the hindfoot during the stance phase of gait. In such circumstances, providing increased dorsiflexion in a staged fashion by any of the means discussed above may be necessary.

\section{Hindfoot correction}

\section{Acute bindfoot correction}

Acute hindfoot correction is most easily achieved with a posterior calcaneal tuberosity osteotomy. Such an osteotomy is created obliquely in a dorsal-proximal to plantar-distal direction. The proximal fragment may then be mobilized medially, laterally, dorsally or plantarward depending upon the deformity correction required. Biplanar correction is facilitated by making the osteotomy more vertical, which allows translation of the posterior segment in both the sagittal and coronal planes. If difficulty is encountered obtaining complete coronal plane correction by translation alone, a closing wedge osteotomy can be performed. This is especially useful when addressing hindfoot varus.

Fixation of the calcaneal osteotomy may be accomplished with partially threaded screws, staples, or fine wire fixation to a circular fixator. Screws must be strategically placed to avoid interference with olive wires utilized for frame stabilization. We have found 4.0 or $4.5 \mathrm{~mm}$ screws helpful to avoid such interference.

\section{Gradual hindfoot correction}

Length of the foot must be considered when planning midfoot and hindfoot deformity correction as many complex deformities, particularly those associated with failed early management of an infantile clubfoot, exist in the setting of a very small stiff foot. When severe cavus and hindfoot varus are present in the setting of a residual clubfoot deformity, combined midfoot dorsal closing wedge and lateral calcaneal closing wedge osteotomies often result in unacceptable shortening of a foot with already compromised length. Gradual correction with or without lengthening of the hindfoot and midfoot segments should be considered in these patients to either maintain existing length or potentially improve foot length (Figure 1). Gradual hindfoot correction may be achieved with a miter frame if the midfoot is to be corrected in concert.

\section{Midfoot correction}

Successful management of midfoot deformity often involves simultaneous correction of sagittal, coronal and axial deformities. Fortunately, gradual correction in a multiplanar external fixator eliminates the vast majority of complexities encountered with acute correction. Gradual correction also allows for correction without loss of foot length and the ability to revise the corrective prescription to modulate correction based upon clinical appearance, radiographic measurements or patient perceptions of residual deformity.

Gradual midfoot correction may be achieved with or without an osteotomy. Regardless of the approach, an open plantar fascia release with striping of the plantar intrinsic musculature from the calcaneus (Steindler stripping) is recommended to achieve as much acute correction as possible and decrease tension across the joints or the osteotomy during correction. Selection of the appropriate multiplanar fixator assembly is determined by the overall deformity correction plan. If ankle and midfoot deformity correction is being performed in a staged fashion, a butt frame following correction of equinus will maintain ankle position while enabling subsequent midfoot correction. If ankle and midfoot deformities are being corrected concurrently, a miter allows for simultaneous correction of both segments or staged correction without the need for frame revision.

Gradual correction without an osteotomy relies on correction through stress relaxation of the capsular and surrounding tissues of the midfoot. This method is more ideal for younger patients with greater tissue compliance and remaining skeletal growth. Correction is maintained, in part, by remodeling of the bones and joints, which is unlikely to occur in skeletally mature patients. Caution should also be exercised in utilizing soft tissue distraction alone in patients with a poorly compliant soft tissue envelope of the foot due to previous surgical intervention and scarring or conditions such as arthrogryposis or diastrophic dysplasia.

Gradual correction through a midfoot osteotomy relies on distraction osteogenesis for maintenance of correction. Distraction through the osteotomy allows for greater surgeon control of the level of correction. The osteotomy site typically is selected as close to the apex of the midfoot 

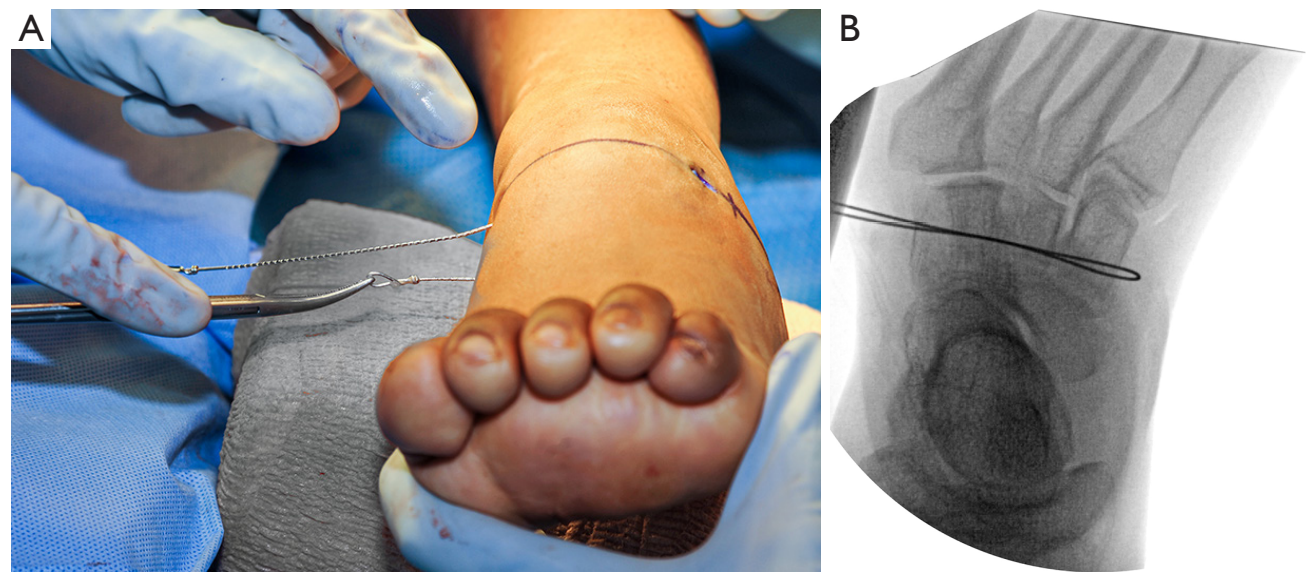

Figure 6 Gigli saw midfoot osteotomy. (A) A Gigli saw is passed percutaneously through four small incisions; (B) intraoperative imaging to assess Gigli saw positioning and confirm the level and orientation of the osteotomy.

deformity as possible. Open and percutaneous osteotomies are both effective though an open osteotomy requires extensive soft tissue dissection through relatively large incisions. A percutaneous osteotomy is easily performed through four small stab incisions to allow passage of a Gigli saw circumferentially about the midfoot between important neurovascular/tendinous structures and the periosteum (Figure 6). Fluoroscopic guidance enables precise placement of the saw to mirror pre-operative planning while avoiding inadvertent intra-articular osteotomies (Figure 6B). This method essentially creates an "invisible osteotomy" that may be difficult to see on plain radiographs or intra-operative fluoroscopy. Furthermore, as the osteotomy results in little to no bone removal and minimal periosteal violation, early consolidation is a concern and surgeons should consider a shorter latency period prior to initiation of correction than would be used following an open osteotomy. Moreover, to mitigate the chance of early consolidation, distraction across the osteotomy site of at least 5 to $10 \mathrm{~mm}$ is advised prior to angular or rotational correction. This also allows for visualization of the osteotomy on subsequent radiographic examinations.

\section{Forefoot considerations}

Though forefoot deformity correction is rarely needed during frame assisted clubfoot reconstructions, it is critical to understand that correction of high grade equinus and/or cavus has the potential to affect a change in toe position as a result of the increased tension on the long toe flexors from relative lengthening of the midfoot and posterior ankle. Severe flexion proximal interphalangeal flexion deformities can result in difficulty with shoe wear and pressure induced pain along the distal digits during weightbearing. This can be a significant source of patient dissatisfaction following an otherwise successful foot reconstruction. To avoid toe deformities, some advocate incorporating the toes into the frame by placing retrograde fine wires across the phalanges into the metatarsals. Wires are then secured to the frame, which maintains toe extension during correction. The preferred method at our institution is to not incorporate the toes into frame and prevent deformity by patient and therapist assisted stretching. Stretching must be performed several times daily but can be extremely effective.

\section{Postoperative considerations}

Once deformity correction has been achieved in the circular fixator we typically stabilize the correction by maintaining the fixator in a static position for an additional 6 weeks. If an osteotomy was performed, healing of the osteotomy site may require a lengthier stabilization period and is monitored on serial radiographs. We permit weight bearing in the fixator during the stabilization period by adding rocker rails to the most distal ring(s). Following frame removal, patients are transitioned to a weight bearing cast for 6 weeks and subsequently transitioned to an $\mathrm{AFO}$ for 6 months. If patients require a tibialis anterior tendon transfer to the lateral cuneiform to maintain deformity correction in the long term, we prefer to perform such a procedure after the resolution of any frame induced osteopenia to avoid tendon transfer failure 
and/or iatrogenic fracture.

\section{Summary}

Circular external fixation is a powerful tool for correcting the segmental and multiplanar foot and ankle deformities present in patients with untreated or recurrent clubfoot deformity. Acute correction may be utilized in combination with gradual distraction across soft tissues, joints, or osteotomies to efficiently correct deformity and minimize soft tissue trauma. Benefits of gradual correction with a circular external fixator include the preservation of foot length and the ability to alter the correction prescription to ensure a plantigrade result. While this technique is effective, patients must be counseled on the treatment duration and possible complications prior to starting treatment. Future research efforts should investigate the effectiveness of minimally invasive soft tissue releases and osteotomies as well the potential benefits of combined acute and gradual correction techniques.

\section{Acknowledgments}

Funding: None.

\section{Footnote}

Provenance and Peer Review: This article was commissioned by the Guest Editors (Federico Canavese and Alain Dimeglio) for the series "Clubfoot" published in Annals of Translational Medicine. The article has undergone external peer review.

Reporting Checklist: The authors have completed the Narrative Review reporting checklist. Available at http:// dx.doi.org/10.21037/atm-20-7621

Peer Review File: Available at http://dx.doi.org/10.21037/ atm-20-7621

Conflicts of Interest: All authors have completed the ICMJE uniform disclosure form (available at http://dx.doi. org/10.21037/atm-20-7621). The series "Clubfoot" was commissioned by the editorial office without any funding or sponsorship. Dr. JRZ reports academic grant to foot and ankle fellowship from Orthofix and reports consulting fees of $\$ 2,000$ from Orthofix. The authors have no other conflicts of interest to declare.
Ethical Statement: The authors are accountable for all aspects of this work and ensure that questions related to the accuracy or integrity of any part of the work are appropriately investigated and resolved.

Open Access Statement: This is an Open Access article distributed in accordance with the Creative Commons Attribution-NonCommercial-NoDerivs 4.0 International License (CC BY-NC-ND 4.0), which permits the noncommercial replication and distribution of the article with the strict proviso that no changes or edits are made and the original work is properly cited (including links to both the formal publication through the relevant DOI and the license). See: https://creativecommons.org/licenses/by-nc-nd/4.0/.

\section{References}

1. Ilizarov GA. The tension-stress effect on the genesis and growth of tissues: Part II. The influence of the rate and frequency of distraction. Clin Orthop Relat Res 1989:263-85.

2. Ilizarov GA. The tension-stress effect on the genesis and growth of tissues. Part I. The influence of stability of fixation and soft-tissue preservation. Clin Orthop Relat Res 1989:249-81.

3. Paley D. The correction of complex foot deformities using Ilizarov's distraction osteotomies. Clin Orthop Relat Res 1993:97-111.

4. Cherkashin AM, Samchukov ML, Birkholts F. Treatment Strategies and Frame Configurations in the Management of Foot and Ankle Deformities. Clin Podiatr Med Surg 2018;35:423-42.

5. Lee DY, Choi IH, Yoo WJ, et al. Application of the Ilizarov technique to the correction of neurologic equinocavovarus foot deformity. Clin Orthop Relat Res 2011;469:860-7.

6. Ferreira RC, Costa MT. Recurrent clubfoot--approach and treatment with external fixation. Foot Ankle Clin 2009;14:435-45.

7. Ferreira RC, Costa MT, Frizzo GG, et al. Correction of severe recurrent clubfoot using a simplified setting of the Ilizarov device. Foot Ankle Int 2007;28:557-68.

8. Hosny GA. Correction of foot deformities by the Ilizarov method without corrective osteotomies or soft tissue release. J Pediatr Orthop B 2002;11:121-8.

9. Bradish CF, Noor S. The Ilizarov method in the management of relapsed club feet. J Bone Joint Surg Br 2000;82:387-91. 
10. Gupta P, Bither N. Ilizarov in relapsed clubfoot: a necessary evil? J Pediatr Orthop B 2013;22:589-94.

11. Lamm BM, Standard SC, Galley IJ, et al. External fixation for the foot and ankle in children. Clin Podiatr Med Surg 2006;23:137-66, ix.

12. Grill F, Franke J. The Ilizarov distractor for the correction of relapsed or neglected clubfoot. J Bone Joint Surg Br 1987;69:593-7.

13. Freedman JA, Watts H, Otsuka NY. The Ilizarov method for the treatment of resistant clubfoot: is it an effective solution? J Pediatr Orthop 2006;26:432-7.

14. Prem H, Zenios M, Farrell R, et al. Soft tissue Ilizarov correction of congenital talipes equinovarus--5 to 10 years postsurgery. J Pediatr Orthop 2007;27:220-4.

15. Waizy H, Windhagen H, Stukenborg-Colsman C, et al. Taylor spatial frame in severe foot deformities using double osteotomy: technical approach and primary results.

Cite this article as: Meyer Z, Zide JR, Cherkashin A, Samchukov M, Bohl DD, Riccio AI. Narrative review of ring fixator management of recurrent club foot deformity. Ann Transl Med 2021;9(13):1104. doi: 10.21037/atm-20-7621
Int Orthop 2011;35:1489-95.

16. Floerkemeier T, Stukenborg-Colsman C, Windhagen $\mathrm{H}$, et al. Correction of severe foot deformities using the Taylor spatial frame. Foot Ankle Int 2011;32:176-82.

17. Eidelman M, Katzman A. Treatment of arthrogrypotic foot deformities with the Taylor Spatial Frame. J Pediatr Orthop 2011;31:429-34.

18. van Gelder JH, van Ruiten AG, Visser JD, et al. Longterm results of the posteromedial release in the treatment of idiopathic clubfoot. J Pediatr Orthop 2010;30:700-4.

19. de la Huerta F. Correction of the neglected clubfoot by the Ilizarov method. Clin Orthop Relat Res 1994:89-93.

20. Wallander H, Hansson G, Tjernstrom B. Correction of persistent clubfoot deformities with the Ilizarov external fixator. Experience in 10 previously operated feet followed for 2-5 years. Acta Orthop Scand 1996;67:283-7. 